\title{
A Case of Recurrent Chalazia Associated with Subclinical Hypothyroidism
}

\author{
Robbert Bipat $^{\mathrm{a}} \quad$ Dineshpersad Jiawan $^{\mathrm{b}} \quad$ Jerry R. Toelsie ${ }^{\mathrm{a}}$ \\ aDepartment of Physiology, Faculty of Medical Science, Anton de Kom University of \\ Suriname, Paramaribo, Suriname; bepartments of Ophthalmology, Academic Hospital \\ Suriname and Faculty of Medical Science, Anton de Kom University of Suriname, \\ Paramaribo, Suriname
}

\section{Keywords}

Chalazion · Subclinical hypothyroidism $\cdot$ Incision and curettage $\cdot$ Levothyroxine

\begin{abstract}
Chalazia are chronic inflammatory disorders of the Meibomian glands of the eyelids that can present at any age. Although the exact cause is still obscure, they are often associated with retention of lipids in these sebaceous glands, infections, or disturbances in the balance of sex hormones. The complications of the disease may vary from little discomfort to reduced vision. Treatment strategies include hot compresses, intralesional steroid injections, and incision and curettage. The present case is about a female in her forties with a 4-year long history of recurrent chalazia. The management of the majority of the lesions was through incision and curettage. Concomitantly she presented with subclinical hypothyroidism, for which she received treatment. During treatment of the hypothyroidism, there was a remission of the chalazia. After cessation of the treatment with levothyroxine, the chalazia started recurring. Reinstatement of a low-dose treatment with levothyroxine eventually led to a remission and prevented further recurrence of the chalazia. Subclinical hypothyroidism may predispose to recurrent chalazia. This finding may have consequences for the understanding of the pathophysiology and the management of this disorder. Further investigations must elicit the exact mechanism of this association.




\section{Case Reports in Ophthalmology}

\section{Introduction}

A chalazion is a common disorder of the eyelids and may present at any age. The disorder is in fact a chronic inflammation of the Meibomian glands in the eyelids, often with resulting granulation [1]. Chalazia are highly prevalent in ophthalmological practice and their rate of recurrence may be up to $35 \%[2,3]$. Most of the time they are the result of retention of lipid material in the sebaceous glands [2]. Other predisposing factors may be viral or bacterial infections of the glands [2]. An association with low vitamin levels as well as disturbed balances of androgens during pregnancy or puberty hints towards metabolic causes [1, 2, 4]. In some cases the disorder is associated with parasitic infestations [5]. Regardless of the cause, they tend to give some degree of discomfort that may vary from just cosmetic disturbance to uncomfortable conjunctivitis or keratitis due to incomplete closure of the eyelids that eventually may lead to spherical aberration of the corneal surface [6].

Histopathologic findings almost always include an obstruction and dilatation of the ducts of the Meibomian glands. Furthermore, the presence of lipid-filled Touton-type giant cells with a foamy appearance of the cytoplasm that reflects the abundance of fat is typical. There is also a varying degree of cells involved with the process of inflammation $[3,7]$.

The initial treatment starts with simple washing of the eyes and hot compresses. Topical antibiotic ointment may be helpful. However, in most cases more invasive procedures like intralesional steroid injections or incision and curettage surgery are necessary [8]. With recurrent variants of the disorder, these latter procedures are inevitable. Whatever the cause, larger lesions must be excised since they may lead to visual aberrations like astigmatism $[6$, 9]. The invasive procedures are not completely innocent and have led to cases of vascular occlusions in the structures of the eye [10] and blindness [11].

The present case involves a female patient presenting with recurrent chalazia and afterwards being associated with subclinical hypothyroidism. Treatment of the hypothyroidism led to a permanent remission of the chalazia.

\section{Case Report/Presentation}

The case involves a then 43-year-old female presenting at the family physician in September 2014 with two painless lumps of about $5 \mathrm{~mm}$ in diameter on both the upper and the lower eyelid of the right eye. Initially, the physician advised her to clean the eyelids thoroughly, avoid application of cosmetic products, and frequently apply hot compresses to the lumps. She also suffered from dry eyes for which he prescribed hypromellose 3\% eye drops. After 1 month, the lumps decreased in size to almost half the initial diameter. However, at this stage they became red and painful. The patient received an antibiotic ointment (tetracycline 1\%). After about 2 weeks, she still suffered from the inflamed lumps. The physician then referred her to an ophthalmic clinic.

The ophthalmologist diagnosed the lesions as chalazia and planned for surgery. On December 12, 2014, he performed the first incision and curettage while he excised the second one similarly on January 12, 2015. The patient recovered without complications. In April 2015 , two other chalazia occurred on the upper lid of the left eye, while a small one presented on a different location of the upper eyelid of the right eye. On July 24, 2015, the ophthalmologist performed the surgical procedure on the chalazia of the left eye. In August 2015, the recent right eye chalazion achieved a size of about $5 \mathrm{~mm}$ and became inflamed. Another chalazion had occurred on the upper left eyelid. Since the patient was due to travel abroad shortly 
after that, it was decided to inject the lumps with triamcinolone $(40 \mathrm{mg} / \mathrm{mL})$. Both lesions regressed in size within 2 weeks.

However, after returning to the country at the end of September, they regained their original size. In October and November 2015, they were excised. By February 2016 there were already two lesions again, and by the end of 2016 she had undergone an additional three surgical procedures to remove chalazia. In December 2016 there were still two lesions in the upper lids of both eyes. The patient was very annoyed at this point and refused further invasive procedures. She decided to leave it as is and to continue with hot compresses, however, without much success.

In a last attempt, the physician performed routine laboratory tests including a lipid profile and evaluation of the reproductive hormones (estrogen, progesterone, luteinizing hormone, follicle-stimulating hormone, prolactin, and testosterone) and thyroid-stimulating hormone (TSH). All results were well within the normal range. However, the TSH was $3.9 \mathrm{mIU} / \mathrm{L}$, which was close to the upper limit. Based on this result he did a full evaluation of the thyroid hormones. These results were $4.2 \mathrm{mIU} / \mathrm{L}(0.4-4.0 \mathrm{mIU} / \mathrm{L})$ for TSH, $70 \mathrm{nmol} / \mathrm{L}$ for thyroxine $\left(\mathrm{T}_{4}\right)$ (70-163 nmol/L), $8 \mathrm{pmol} / \mathrm{L}$ for free $\mathrm{T}_{4}\left(\mathrm{fT}_{4} ; 10-24 \mathrm{pmol} / \mathrm{L}\right)$, and $1.0 \mathrm{nmol} / \mathrm{L}$ for tri-iodothyronine $\left(\mathrm{T}_{3}\right)(1.1-2.6 \mathrm{nmol} / \mathrm{L})$.

There were no clinical signs of hypothyroidism. She was not overtly obese $(157 \mathrm{~cm}$ in height and $59 \mathrm{~kg}$ in weight) and the resting heart rate varied from 70 to 90 beats/min. In general, she always felt well, had a good appetite with regular stools, and a menstrual cycle of $28 \pm 2$ days. Suspecting a borderline subclinical hypothyroidism, the physician started treatment with a low dose of levothyroxine ( $6.25 \mu \mathrm{g} /$ day) and gradually increased it to $12.5 \mu \mathrm{g} /$ day within the span of 3 weeks. The patient did not show any adverse reactions to this treatment. Remarkably, both the original chalazia and a third one that developed in the meantime in the upper left eyelid gradually reduced in size. Concomitantly, the inflammation also disappeared and 4 months later, in August 2017, they had all vanished. No new chalazia had developed and the dry eyes were no longer an issue. TSH reduced to $1.4 \mathrm{mIU} / \mathrm{L}, \mathrm{T}_{4}, \mathrm{fT}_{4}$, and $\mathrm{T}_{3}$ increased to $86 \mathrm{nmol} / \mathrm{L}, 14 \mathrm{pmol} / \mathrm{L}$, and $1.3 \mathrm{nmol} / \mathrm{L}$, respectively.

In January 2018, the physician decided to taper off the treatment in the course of 2 months under monitoring of the thyroid hormones. In April 2018, after cessation of the levothyroxine supplementation, a lump started to grow in the lower right eyelid. Laboratory values revealed again borderline values for the hormones $\mathrm{T}_{4}(80 \mathrm{nmol} / \mathrm{L}), \mathrm{fT}_{4}(12 \mathrm{pmol} / \mathrm{L})$, and $\mathrm{T}_{3}(1.0 \mathrm{nmol} / \mathrm{L})$. TSH was within the normal range at $2.0 \mathrm{mIU} / \mathrm{L}$. After 2 months, the lesion started to grow quickly and another one was developing in the lower left eyelid. In July 2018, treatment with levothyroxine was restarted at $12.5 \mu \mathrm{g} /$ day. By October, both lumps had disappeared completely. From January 2019 the dose of levothyroxine was reduced to 6.25 $\mu \mathrm{g} /$ day. No new chalazia occurred up to October 2019 . The patient remains on a low-dose regimen of thyroxine.

\section{Discussion/Conclusion}

This case concerns a female patient in her forties with recurrent chalazia over the course of 4 years. The majority of the lesions were removed by incisions and curettage. By serendipity, it was eventually found that the chalazia were associated with a borderline low thyroid hormone level. In the end, a low-dose regimen of levothyroxine prevented the recurrence of chalazia for at least a year. To our knowledge, this is the first time that this association was found in a patient. 
A possible explanation for this observation may be the fact that $\mathrm{T}_{3}$ hormone reduces plasma lipoprotein levels [12] and consequently can prevent accumulation of these lipid materials in the Meibomian glands. In addition, earlier studies showed a direct relation between thyroid hormone levels and the secretion of sebaceous glands in rats [13] and humans [14]. In a population study, researchers found a significant association between hypothyroidism and blepharitis [15], a condition that can be a prelude to chalazia [16]. Another earlier report mentioned cases of severe hypothyroidism that presented with loss of eyelashes; however, none of them developed chalazia [17]. No other reports on this subject are readily available and on the contrary, cases of hyperthyroidism are associated with chalazia as a result of blepharitis that occurs due to the exophthalmia [18].

The present findings give rise to reconsideration of the etiology of the relatively common disorder of chalazion and should trigger us to evaluate the endocrine profile of patients with recurrent variants of this disorder. In addition, we must reconsider the range of thyroid hormones in the context of clinical presentations. It should also be recommended that a long-term follow-up of these patients may be necessary. Future research on the exact mechanism of action is warranted.

\section{Statement of Ethics}

The patient gave oral and written consent to publish the data. The report does not include personal information that could identify the patient directly or indirectly. All medical interventions have been carried out according to the latest protocols of therapy. Reporting and writing are all in compliance with the Declaration of Helsinki.

\section{Disclosure Statement}

The authors have no conflicts of interest to declare.

\section{Funding Sources}

Robbert Bipat and Jerry R. Toelsie are employed by the Anton de Kom University of Suriname and are both part-time physicians. They receive a monthly payment from the University and the Sate Medical Insurance company. Dinesh Jiawan is employed by the Academic Hospital of Paramaribo in Suriname and receives his monthly fee from this institution. No other funding resources are connected to this study.

\section{Author Contributions}

Robbert Bipat combined the data and designed, drafted, and finalized the manuscript. Dinesh Jiawan performed the surgery, conducted the follow-up of the patient, and critically reviewed the manuscript. Jerry R. Toelsie collected the laboratory data, performed the medical treatment, and critically reviewed the manuscript. All authors approved the final version of the manuscript and are accountable for all aspects of the work. All authors attest that they meet the current ICMJE criteria for authorship. 


\section{References}

1 Arbabi EM, Kelly RJ, Carrim ZI. Chalazion. BMJ. 2010 Aug;341:c4044.

2 Alsammahi A, Aljohani Z, Jaad N, Abu Daia O, Aldayhum M, Almutairi M, et al. Incidence and predisposing factors of chalazion. Int J Community Med Public Health. 2018 Oct;5(11):4979.

3 Özdal PÇ, Codère F, Callejo S, Caissie AL, Burnier MN. Accuracy of the clinical diagnosis of chalazion. Eye (Lond). 2004 Feb;18(2):135-8.

4 Chen L, Chen X, Xiang Q, Zheng Y, Pi L, Liu Q, et al. Prevalence of low serum vitamin a levels in young children with chalazia in Southwest China. Am J Ophthalmol. 2014 May 1;157(5):1103-08.e2.

5 Schear MJ, Milman T, Steiner T, Shih C, Udell IJ, Steiner A. The Association of Demodex with Chalazia: A Histopathologic Study of the Eyelid. Ophthal Plast Reconstr Surg. 2016 Jul-Aug;32(4):275-8.

6 Park YM, Lee JS. The effects of chalazion excision on corneal surface aberrations. Cont Lens Anterior Eye. 2014 Oct;37(5):342-5.

7 Gutgesell VJ, Stern GA, Hood CI. Histopathology of meibomian gland dysfunction. Am J Ophthalmol. 1982 Sep;94(3):383-7.

8 Goawalla A, Lee V. A prospective randomized treatment study comparing three treatment options for chalazia: triamcinolone acetonide injections, incision and curettage and treatment with hot compresses. Clin Exp Ophthalmol. 2007 Nov;35(8):706-12.

9 Jin KW, Shin YJ, Hyon JY. Effects of chalazia on corneal astigmatism : large-sized chalazia in middle upper eyelids compress the cornea and induce the corneal astigmatism. BMC Ophthalmol. 2017 Mar;17(1):36.

10 Thomas EL, Laborde RP. Retinal and choroidal vascular occlusion following intralesional corticosteroid injection of a chalazion. Ophthalmology. 1986 Mar;93(3):405-7.

11 Hoşal BM, Zilelioğlu G. Ocular complication of intralesional corticosteroid injection of a chalazion [Internet]. Eur J Ophthalmol. 2003 Nov-Dec;13(9-10):798-9. [cited 2019 Dec 2].

12 Hansson P, Valdemarsson S, Nilsson-Ehle P. Experimental hyperthyroidism in man: effects on plasma lipoproteins, lipoprotein lipase and hepatic lipase. Horm Metab Res. 1983 Sep;15(9):449-52.

13 Thody AJ, Shuster S. A study of the relationship between the thyroid gland and sebum secretion in the rat. J Endocrinol. 1972 Aug;54(2):239-44.

14 Goolamali SK, Evered D, Shuster S. Thyroid disease and sebaceous function. BMJ. 1976 Feb;1(6007):432-3.

15 Nemet AY, Vinker S, Kaiserman I. Associated morbidity of blepharitis. Ophthalmology. 2011 Jun;118(6):1062-8.

16 Nemet AY, Vinker S, Kaiserman I. Associated morbidity of chalazia [Internet]. Cornea. 2011 Dec;30(12):1376-81. [cited 2019 Dec 4].

17 Mahto RS. Ocular features of hypothyroidism. Br J Ophthalmol. 1972 Jul;56(7):546-9.

18 Bernardes TF, Bonfioli AA. Blepharitis. Semin Ophthalmol. 2010 May;25(3):79-83. 